\title{
Impregnated Material Dosage Form Category
}

National Cancer Institute

\section{Source}

National Cancer Institute. Impregnated Material Dosage Form Category. NCI Thesaurus. Code C149570.

A type of solid pharmaceutical dose form consisting of a material onto or into which active substance(s) are absorbed for subsequent release after administration, often over an extended period of time. 